\title{
Mean Field Modeling of the FitzHugh-Nagumo Neuronal Network Model with Kernel Functions and Time-Delayed Couplings
}

\author{
Algis GARLIAUSKAS \\ Vilnius University, Institute of Mathematics and Informatics \\ Akademijos 4, LT-06883 Vilnius, Lithuania \\ e-mail:algis.garliauskas@vu.mii.lt
}

Received: September 2011; accepted: March 2013

\begin{abstract}
In this paper, the nonlinear neural network FitzHugh-Nagumo model with an expansion by the excited neuronal kernel function has been investigated. The mean field approximation of neuronal potentials and recovery currents inside neuron ensembles was used. The biologically more realistic nonlinear sodium ionic current-voltage characteristic and kernel functions were applied. A possibility to present the nonlinear integral differential equations with kernel functions under the Fourier transformation by partial differential equations allows us to overcome the analytical and numerical modeling difficulties. An equivalence of two kinds solutions was confirmed basing on the errors analysis. The approach of the equivalent partial differential equations was successfully employed to solve the system with the heterogeneous synaptic functions as well as the FitzHugh-Nagumo nonlinear time-delayed differential equations in the case of the Hopf bifurcation and stability of stationary states. The analytical studies are corroborated by many numerical modeling experiments.

The digital simulation at the transient and steady-state conditions was carried out by using finite difference technique. The comparison of the simulation results revealed that some of the calculated parameters, i.e. response and sensitivity is the same, while the others, i.e. half-time of the steadystate is significantly different for distinct models.
\end{abstract}

Key words: neural network, mean field approximation, FitzHugh-Nagumo differential equations, bifurcation, stability.

\section{Introduction}

The brain consisting of multi-massive structure in general and especially of the multineural organization for humans and some primates brain is laid out as clustered higher and lower cell density mosaics. The ordered structures are probably formed by self-organizing mechanisms (Cowan, 1982; Garliauskas, 2003). The brain, created by neuronal networks, the properties of which are expressed by nonlinear, excitation/inhibition potential fields, signal delays, stochastic, is a very complex system for modeling and control analysis. The complexity is aggravated having in mind noises, nonlinearities, space and time distribution. They take place in neuronal system elements: synapses, dendrite membranes, soma of 
neurons, axons. The mostly complicated characteristic is the sodium ionic current-voltage $\mathrm{N}$-shaped relation of synapses (Garliauskas, 1998). Beside, when considering neuronal networks of the brain the synaptic couplings among interconnected neurons acquire an important biological meaning and modeling presumption. The intensity or strength of couplings is distributed in the space and represented, as a rule, by the kernel function. The kernel function can reflect pure excitations, lateral inhibitions (the so-called Mexican hat kernel function) or lateral excitations (Zhang et al., 2010). The lateral inhibition function is more surprising and implies that neurons close to one another have excitatory connections and neurons faraway have inhibitory ones.

Neuronal dynamics and stability of the differential equations described have been solved in wide aspects, beginning with Hodgkin and Huxley (1952), FitzHugh (1961) and Nagumo et al. (1962), Mackey and Nechaeva (1995), Zhang et al. (2010) and finishing with Tanabe and Pakdaman (2001), Hasegawa (2003, 2004). Hasegawa solves the dynamics of the FitzHugh-Nagumo model of neuron ensembles with time-delayed couplings among neurons, noises and stochastic. Tanabe considers the solutions by numerical calculations for single Hodgkin-Huxley neurons. Zhang studies the traveling wave fronts in synaptic coupled neuronal networks more from the mathematical point of view.

Our goal in difference to others is to study the influence of nonlinear coupling kernel functions and other strong nonlinearities on searching of the neuronal dynamics solutions, stability problems, though, in the first stage, restricting to a mean field approximation.

The paper is organized as follows. In Section 2, the biological presumptions and and more realistic models are discussed. The presumptions of simplifications and the mathematical modeling of the coupling neuronal network dynamics are studied in Section 3. Section 4 is devoted to study of employing to solve the system with the heterogeneous synaptic functions. The solution of the FitzHugh nonlinear time-delayed differential equations of the Hopf bifurcation point of view and its proofs are proposed in Section 5 and Appendix A. Finally, Section 7 concludes the paper by giving a resume of some direction for a further investigation.

\section{The Biological Presumptions and Models}

The cortex of the brain is super multiplex neuronal system. In reality the cortex can be approximated as a three-dimensional layer, about $3 \mathrm{~mm}$ thick and nearly $84 \mathrm{~cm}^{2}$ in the surface area. It has been estimated by neurophysiologists that there are nearly 300 differing neuron-cell types (Cowan, 1982). However, almost all types of neurons have one main exclusive property - the threshold voltage characteristic. All neurons together with other compartments (synapses, dendrites and axons) compose a synapse-dendrite-soma-axon chain with distributed electrical and biochemical parameters on the cortex surface (Szentagothai, 1974). It is necessary to emphasize that there are exist essential nonlinearities, signal delays, and stochastic expressed by noises. All chains are structured in the neuronal networks with specialized functional neurophysiological contributions.

Consider the main characteristics of the synapse-dendrite-soma-axon chain. The synapse behavior has theoretically corroborated that the stead inward sodium current, 


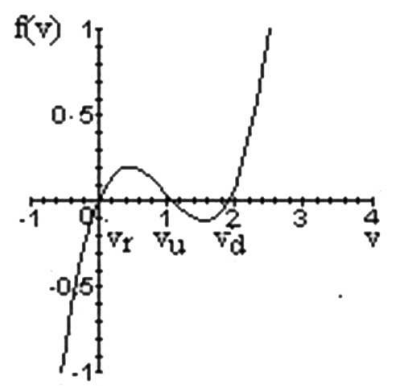

Fig. 1. The sodium current voltage characteristic.

related to voltage, reflecting a nonlinear process of activation, had slopes of positive and negative conductance arising from the supposed existence of the N-shaped currentvoltage characteristic (Garliauskas, 1998; Schwindt and Crill, 1977). This characteristic, shifted the rest potential to the beginning coordinates, is approximated by the polynomial expression of a dimensionless type as follows

$$
f(v)=v+\alpha v\left(v^{2}-3 v\right)
$$

where non-dimensional constant $\alpha=0.48$. Some authors (Zhang, 2010; Hasegawa, 2004) use the linear function $f_{0}(v)=a v$, where $a$ is as a conductance. The characteristic curve crosses the abscissa at three points $v_{r}, v_{u}$ and $v_{d}$ (Fig. 1). A segment is stable in the area around the point $v_{r}$ around the point $v_{u}$ is an unstable while a stable depolarized segment of the firing state is close to the point $v_{d}$.

Biologically a cubic function (1) is different in principle and more adequate than a linear one to model neuronal networks used, for example, in Zhang (2010).

The couplings between neurons in the neural network may be expressed as a pure excitation, lateral inhibition or lateral excitation. These main characteristics inside neural networks in the space of the cortex are formed by the kernel function which may be a one-dimensional or two-dimensional function. The mathematical approximations are as follows

$$
K(x)=\left(\frac{2}{\sqrt{3}} \pi^{-1 / 4}\right)\left(1-x^{2}\right) e^{-\frac{1}{2} x^{2}}
$$

and

$$
K(x, y)=\left(1-\left(x^{2}+y^{2}\right)\right) e^{-\frac{\alpha\left(x^{2}+y^{2}\right)}{2}},
$$

respectively. Graphically they are shown in Figs. 2(a) and 2(b) at $\alpha=0.2$.

The continuum limit is achieved by fixing not only the activity of separate neurons but also the given volume of neuronal tissue centered at a point $x$ or $(x, y)$ in the twodimensional case. 

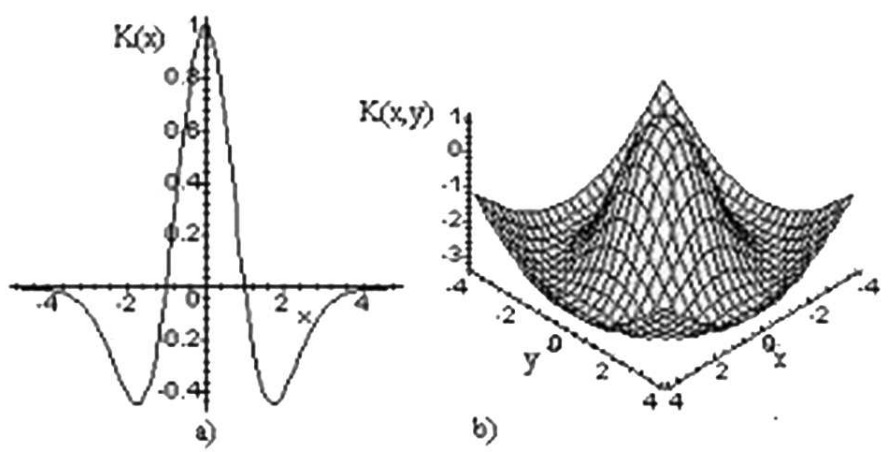

Fig. 2. The kernel functions: (a) the one-dimensional kernel function, (b) the two-dimensional kernel function.

A neuron cell body (soma) is the central part of the neuronal chain which on by means of biochemical and electrical processes maintains the life of cell. The neuron produces the summation of the spatial and temporal electrical signals through dendrites and axons disregarding a biochemical enzymatic mechanism of the neuron cell body. If the summation signal overcomes the axon hillock's threshold, then the neuron fires and the output signal is transmitted along axon to other neurons. A space distribution of state parameters in dendrites and axons is not considered applying a fixed point statement.

The model of a neuron, as a rule, is presented in general by a squashing non-decreasing function

$$
\lim _{v \rightarrow+\infty} g(v)=1, \quad \lim _{v \rightarrow-\infty} g(v)=-1 .
$$

The individual squashing function is a signum function without threshold or with one

$$
\operatorname{sgn} g(v)= \begin{cases}1, & \text { if } v \geqslant 0 \\ -1, & \text { if } v<0\end{cases}
$$

or Heaviside step function with a threshold $h: H(v-h)=0$ for all $v<0$ and $H(v-h)=1$ for all $v \geqslant 0$. Another threshold function that we use is the sigmoid continuum function

$$
g_{i}(v)=\tanh \left(\sum_{j} w_{i j} g\left(v_{j}-h\right) / T\right)
$$

where the sum expresses the common firing potentials from all $j$-side neurons in $i$-side of the neuronal network, $T$ is a temperature which characterizes the noise properties.

These neural cell functions will be used in the modeling bellow. 


\section{Mathematical Model of the Coupling Neuronal Networks}

\subsection{Presumptions of Simplifications}

It has been mentioned above that the different types of neuron cells achieve hundreds below each point on the cortical surface. The one type of neuron is characterized as one degree of freedom from the analytical point of view. Thus, the analytical problem is not solvable. However, Hubel and Wiesel (1962) have discovered that almost all neuron cells under a given point on the cortical surface have the same spatial orientation representing locally by about ten groups of cells involving ten degrees of freedom. But even after such a simplification the analysis remains a formidable task. As it has been shown in Iooss and Joseph (1980) the problem is not so bad because the emergence of patterns of coherent activity of neurons may be controlled by a single maximal eigenvalue of differential equations that describe neuronal dynamics. Therefore the analytical task can be reduced by involving at most one or two degrees of freedom (Iooss and Joseph, 1980).

Further, we are going to use the mean field approximation of the neuronal potentials and recovery current inside the neuron ensembles. We shell first suppose that: (a) the distribution of the above-mentioned state variables is Gaussian and (b) large number of neurons in the ensemble allow us to present the local random variables as the expectation with respect to the corresponding distribution according to the law of large numbers (Hale and Lamel, 1993). In the mean field approximation approach it is assumed that the condition (b) is approximately true even for a finite but large number of neurons.

\subsection{The Mathematical Model of Neuronal Dynamics}

According to mean field approximation the continuum limit will be obtained by estimating, not separate potentials of individual neurons but on their amount proportion becoming active per unit time in given volume elements of neuron ensemble centered at the point. A dynamic evolution of the action of the neuron ensemble consisting of $N$ FitzHughNagumo neurons in the cortical space kernel distribution of potentials on the surface, noise, delaying time, and applied inputs in general is described by the $2 N$-dimensional nonlinear stochastic integral differential evolution equations given by

$$
\begin{aligned}
\epsilon \frac{\partial v_{i}(x, t)}{\partial t}= & f\left[v_{i}(x, t)\right]-e w_{i}(x, t)+\frac{1}{N-1} \iint K(y, z) \\
& \times \sum_{j=1(j \neq i)} N \mu_{i j} g\left[v_{j}\left(t-\tau_{i j}-h\right)\right] d y d z+\xi_{i}(t)+I(t), \\
\frac{\partial w_{i}(x, t)}{\partial t}= & a v_{i}(x, t)-b w_{i}(x, t), \quad i=1,2, \ldots, N,
\end{aligned}
$$

where $f\left[v_{i}(x, t)\right]$ expresses by (1) independent of $x$ and $t, K(y, z)$ is determined by (3) or in one-dimensional case by (2) changing of coordinate names, $g[\cdot]$ by $(6), w_{i}(x, t)$ is the recovery potential function, $\mu_{i j}$ and $\tau_{i j}$ are coupling strength and delay, respectively, 
which in the uniform couplings $\mu_{i j}=\mu$ and delay times $\tau_{i j}=\tau$, and $\epsilon, a, b, e$ are constants. The fourth term of Eq. (7), $\xi_{i}(t)$ denotes the Gaussian white noise. The fifth term in the same equation, $I(t)$, expresses an external input current.

According to the above given mean field approximation presumption, the global variables for the neuron ensemble are almost identical neurons given by

$$
\begin{aligned}
& V(x, t)=\frac{1}{N} \sum_{i=1}^{N} v_{i}(x, t), \\
& W(x, t)=\frac{1}{N} \sum_{i=1}^{N} w_{j}(x, t),
\end{aligned}
$$

Now the function $f\left[v_{i}(x, t)\right]$ becomes $f[V(x, t)]$. The function $g\left[V_{j}(x,(t-\tau))-h\right]$ in (7) applying sigmoid function $g\left[V_{j}(x,(t-\tau))-h\right]=\tanh \left[V_{j}(x,(t-\tau))-h\right]$ and linearizing by Taylor expansion the term $\frac{1}{N} \sum_{j}^{N} \mu g\left[V_{j}(x,(t-\tau))-h\right]=\mu V(x,(t-$ $\tau))-h$.

Assuming that the local variables can be represented as the global variables, onedimensional kernel function $(2), \xi(t)$ as a stationary, independent of space Gaussian white noise, process with $M\{\xi(t)\}=0$ (expectation function) and covariance functions $m\{\xi(t) \xi(s))\}=\delta(t-s)$ (the Dirac delta function), $I(t)=0$, and so far without considering the recovery equation, the dynamic process will be presented by the scalar integral differential equation with two-dimensional couping function $K(y, z)$ as follows

$$
\left.\epsilon \frac{\partial V(x, t)}{\partial t}=f[V(x, t)]+\mu \int_{R} \int_{R} K(y, z) V(x,(t-\tau))-h\right) d y d z .
$$

The solution of Eq. (11) with a convolution product under the integral is more complicated than without it. To simplify finding the solution of Eq. (11) there is a way to transform the integral differential equation to many particular partial differential equations if to apply the Fourier transformation (FT) of the kernel function and the convolution product. The authors (Laing and Troy, 2003; Zhang et al., 2010) propose an appropriate method of transformation.

Let us use the direct FT and the inverse one of the one-dimensional kernel function written as follows

$$
\begin{aligned}
& \hat{K}(\psi)=\int_{R} K(z) \exp (-i z \psi) d z, \\
& K(z)=\frac{1}{2 \pi} \int_{R} K(\psi) \exp (i z \psi) d \psi,
\end{aligned}
$$

respectively.

Applying the Fourier transformation to the Eq. (11) we deal with the convolution product, where the direct FT of functions product equal to product of FT individual functions, 
i.e., $\{f(\psi) * g(\psi)\}=\{f(\psi)\}\{g(\psi)\}$, where $\{*\}$ means the FT of appropriate functions Then Eq. (11) for images will be presented as

$$
\hat{V}_{t}(\psi \cdot t)=\hat{f}[V(\psi, t)]+\hat{K}(\psi) \hat{H}(V(\psi, t)-h),
$$

where Eq. (13) is written without delay ( $\tau=0$ ), the wide hat above functions denotes the direct Fourier transformation. The direct FT of the Heaviside $H$ function is the same function. If and only if $\hat{K}(\psi)$ can be represented by $\frac{1}{K(\psi)}$, where $K(\psi)$ will be polynomial function of $\hat{K}(\psi)$ of $\psi$, then it is possible Eq. (13) applying the inverse FT to obtain the equivalent partial differential equation. Taking in account the simplified form of the kernel function the direct FT

$$
\hat{K}(\psi)=\frac{\rho^{2}}{\rho^{2}+\psi^{2}}
$$

instead of (2) and its inverse FT

$$
K(z)=\frac{\rho}{2} \exp (-\rho|z|),
$$

where $\rho$ is a constant, and using formula (from Laing and Troy, 2003; Zhang et al., 2010)

$$
\sum_{m=0}^{\infty} \frac{(-1)^{m}}{m !(4 \rho)^{m}} \frac{\partial^{2 m}}{\partial x^{2 m}}\left[\frac{\partial V(x, t)}{\partial t}-f[V(x, t)]\right]=H(V(x, t)-h)
$$

the equivalent partial differential equation will be as follows

$$
\begin{aligned}
& \frac{\partial V(x, t)}{\partial t}+f[V(x, t)] \\
& \quad-\frac{1}{4 \rho}\left[\frac{\partial^{3} V(x, t)}{\partial x^{2} \partial t}+\left(\frac{\partial^{2} f[V(x, t)]}{\partial V^{2}}+\frac{\partial f[V(x, t)]}{\partial V}\right) \frac{\partial^{2} V(x, t)}{\partial x^{2}}\right] \\
& =H(V(x, t)-h) .
\end{aligned}
$$

\subsubsection{Numerical Results}

To confirm the equivalence of a direct integral differential solution and that of an equivalent partial differential equation it was fulfilled a numerical modeling both of Eq. (11) in one-dimensional case and (14) in two-dimensional one based on Maple 13 toolkit.

To lead the numerical modeling initial and boundary conditions were given for (11)

$$
I B C=\left\{V(x, 0)=\frac{\rho}{2} \exp (-\rho|x|), V(10, t)=0.0\right\}
$$

and for (14)

$$
I B C^{\prime}=\left\{V(x, 0)=\frac{\rho}{2} \exp (-\rho|x|), V(10, t)=0.0, V(-10, t)=0.0\right\} .
$$



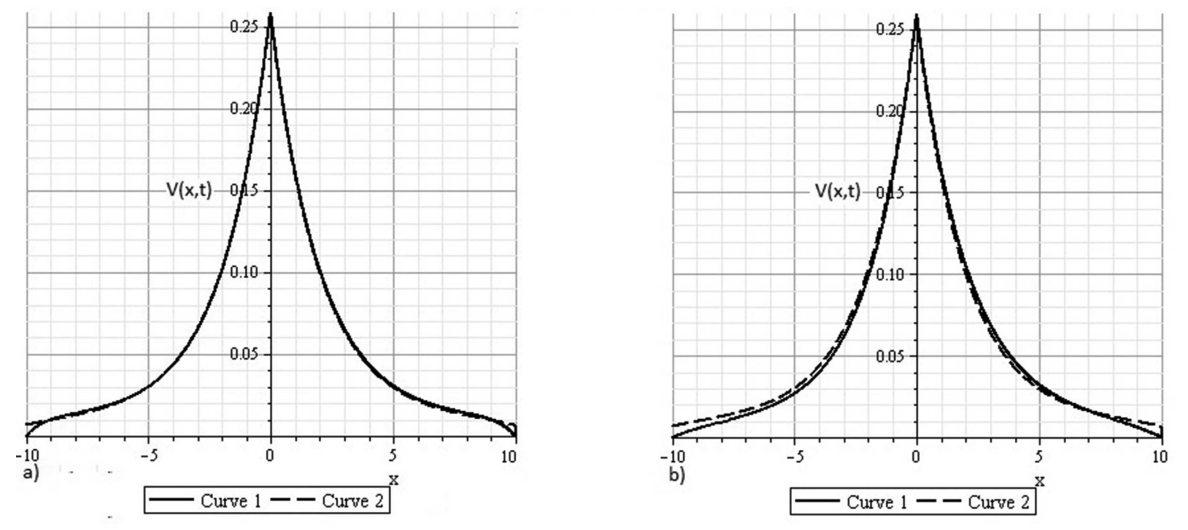

Fig. 3. Spatial solutions at fixed time: (a) (1) the direct equation solution (solid line) and (2) the solution of equivalent partial differential equation (dashed line) at $t^{*}=0.05$ are shown. (b) the same at $t^{*}=0.1$.
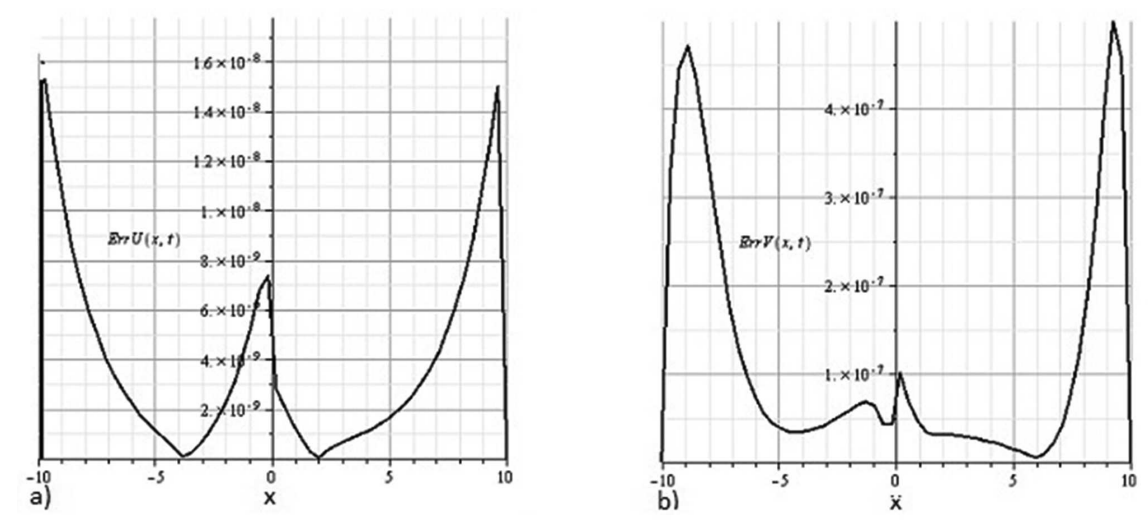

Fig. 4. The local error estimate dependent on the space coordinate and fixed time. The curve of spatial error estimate dependent on the space coordinate for the direct solution at fixed time $\left(t^{*}=0.02\right)$ (a), and the curve of the spatial error estimate at fixed time $\left(t^{*}=0.4\right)$ for the partial equation solution (b) are presented.

The modeling was fulfilled giving the bipolar Heaviside function of type $1-2 H(V(x, t)-$ $h$ ) with threshold $h=2.5$. The calculations were leaded in dimensionless case of variables and parameters. The comparison curves of the direct solution and equivalent one are shown in Figs. 3(a), 3(b) at $t^{*}=0.05$ and $t^{*}=0.1$, respectively. The deviation of the solution curves is challenged because the direct numeric integration was done in a restricted range and with one boundary condition and the partial differential equation was solved with two boundary conditions.

The spatial error estimates for the fixed time in the case of the direct (Fig. 4(a)) and partial differential equation solution (Fig. 4(b)) show that maximum errors are determined near to the marginal boundary points and where there is a sharp peak of the initial condition. The errors are quite small and thus the numerical modeling confirms the equivalence of the direct integral differential solution and that of the partial differential equation (PDE). 

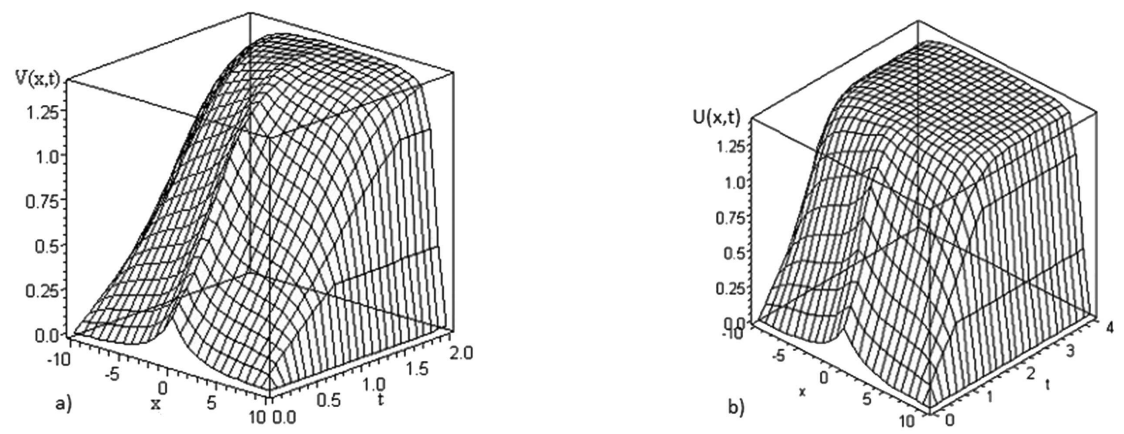

Fig. 5. The solutions of the direct (a) and partial differential equation (b) in three-dimensional space.

The equivalence of the dynamical solutions as well as their stability are testified by the 3D pictures in Figs. 5(a), 5(b).

\section{Solution of the PDE System with a Recovery Factor}

\subsection{Description of the Neural Network PDE System}

Let us consider a neural network system with a recovery current of neuron and including the synaptic coupling thresholds. A mathematical model is presented by the PDE system.

$$
\begin{aligned}
\epsilon \frac{\partial v_{i}(x, y, t)}{\partial t}= & -\alpha v_{i}(x, y, t)+f_{i}\left[v_{i}(x, y, t)\right]-k u_{i}(x, y, t) \frac{1}{N-1} \\
& \times \int_{R^{2}} \int K(x, y) H\left\{\mu_{i j} \sum_{j=1(j \neq i)}^{N} f_{j}\left[v_{j}(x, y, t)-h_{j}\right]\right\} d x d y \\
\nu \frac{\partial u_{i}(x, y, t)}{\partial t}= & d v_{i}(x, y, t)-e u_{i}(x, y, t), \quad i, j=2, \ldots, N
\end{aligned}
$$

where the two-dimensional coupling function

$$
K(x, y)=\frac{k_{1}}{2 \pi \sigma^{2}} \exp \left(-\frac{1}{2 \sigma^{2}}\left[(x-r)^{2}+(y-s)^{2}\right]\right),
$$

$\alpha$ is a decay coefficient, $d, e, k, k_{1}, \sigma, \epsilon, v$ are constants. The PDE system (16), (17) is justified only if $N \geqslant 2$.

\subsection{Numerical Modeling of the System with the Heterogeneous Synaptic Functions}

System (16), (17) was solved at a fixed point of coordinates on the cortex surface, for example, $(x, y)=(1,1)$ by Maple 12 toolkit for an ordinary differential equation (ODE) 

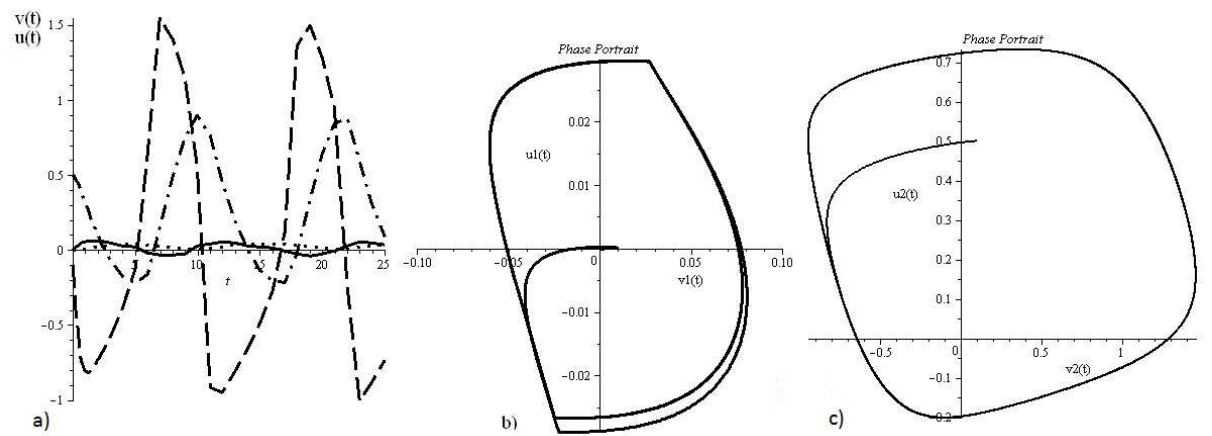

Fig. 6. The neuronal dynamics with the spatial coordinates fixed. The parameters are $\alpha=0.05, k=2, \mu=0.1$, $h=2.5, d=0.2, c=0.1, \epsilon=1, v=1$. (a) $u_{1}(t)$ is the solid curve, the $v_{1}(t)$ is dotted one, $u_{2}(t)$ is the the dash-dotted one, and the $v_{2}(t)$ is long-dashed one. (b) the phase portrait is shown in the case of a bipolar Heaviside function for functions $v_{1}(t), u_{1}(t)$. (c) shows the phase portrait at the unipolar Heaviside function for $v_{2}(t)$, $u_{2}(t)$.

$2 \mathrm{~N}$ system. The ODE system for identical descriptions of $f, \mu, h$ is as follows

$$
\begin{aligned}
\epsilon \frac{d v_{i}(t)}{d t}= & -\alpha v_{i}(t)+f_{i}\left[v_{i}(t)\right]-k u_{i}(t) \\
& +\frac{1}{N-1} \iint_{R^{2}} K(x, y) H\left\{\mu \sum_{j=1(j \neq i)}^{N} f_{j}\left[v_{j}(t)-h\right]\right\} d x d y, \\
\nu \frac{d u_{i}(t)}{d t}= & d v_{i}(t)-e u_{i}(t), \quad i, j=1, \ldots, N,
\end{aligned}
$$

where $R^{2}$ is a real number two-dimensional space.

Calculus Example. The calculus was made including different synaptic functions: $f_{1}\left[v_{1}(t)\right]$ as $(1)$ and $f_{2}\left[v_{2}(t)\right]=v_{2}(t)-v_{2}(t)^{2}\left(v_{2}(t)-1\right)$. A neural network was taken for $N=2$. The modeling results given with the initial conditions $v_{1}(0)=0, u_{1}(0)=0$, $v_{2}(0)=-0.1, u_{2}(0)=0.5$ by Maple dsolve and phase portrait procedures are shown in Fig. 6. Figure 6(a) illustrates the time courses of four independent variables including two excitatory or spiking and two recovery functions. The curves testify about periodic stable oscillations. The phase portrait in Fig. 6(b) confirms that showing the limit circle with the stairs formed by stepwise nonlinearities of synaptic and neuronal action functions.

\section{The Solution of FitzHugh-Nagumo Equations with Kernel Function}

\subsection{Modeling of Dynamics}

The FitzHugh-Nagumo model we expose including space potential distributions - kernel functions that influence the dynamic solution. The model consists of two integral differ- 


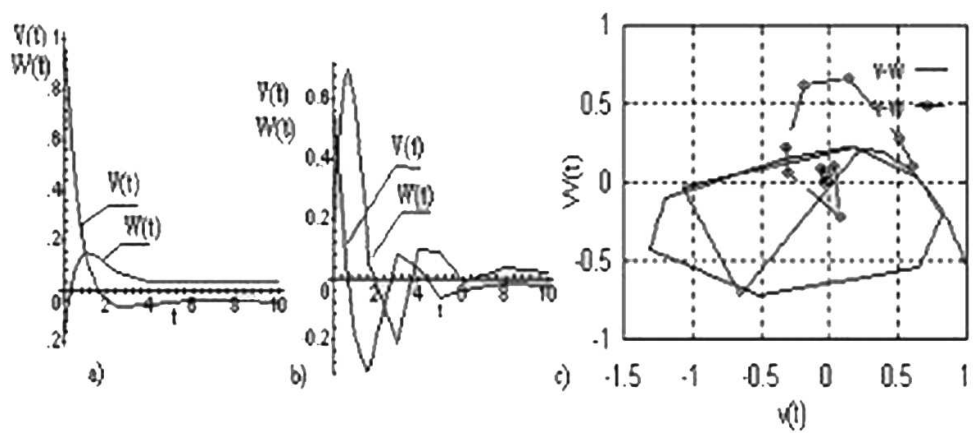

Fig. 7. The potential and recovery currency solutions versus time: (a) the time courses without oscillations, (b) the courses with quenched oscillations, and (c) the phase portraits.

ential equations formulated to mean field of excitation and recovery potential functions and decay term looks like

$$
\begin{aligned}
\epsilon \frac{\partial V(t)}{\partial t}= & -(c+1) V(t)-U(t)+d V(t)\left(V(t)^{2}-V(t)\right) \\
& +\mu \iint\left(1-(x-r)^{2}+(y-s)^{2}\right) e^{-\frac{\left((x-r)^{2}+(y-s)^{2}\right)}{2}} V(t) d r d s, \\
\frac{\partial U(t)}{\partial t}= & a V(t)-b U(t),
\end{aligned}
$$

where $f(V)=-V+d V\left(V^{2}-V\right)$ is shifted to the left comparing with (1), i.e., more realistic. The main influence of the space parameters is expressed by convolution product under the integral sign.

The modeling results of the model presented by Eqs. (18), (19) at initial conditions $V(0)=1, U(0)=-0.2$, coefficients $a=0.14, b=0.4, c=0.1, d=1.098, \mu=0.2$, and $\epsilon=1.0$ in the space fixed $(x, y)=1$ are shown in Fig. 7(a) and at $V(0)=0.6, U(0)=0.2$ in Fig. 7(b). A behavior of the solutions depends on values of parameters. It is reflected by time course without oscillations (Fig. 7(a)) and with ones (Fig. 7(b)). The phase portraits with interpolation numeric results are illustrated in Fig. 7(c), where the portrait of Fig. 7(b) results denoted by line-points curve driven to local focus and the portrait marked by line curve driven to the periodic circle. In last case, the modeling was done at other parameters than Fig. 7(a).

\subsection{Estimation of Stability and Bifurcation}

The analysis of a stability and bifurcation with degeneracy of neuronal networks better to do basing on a standard linearizing approach of the evolutionary differential equations including delay

$$
\epsilon \frac{\partial V}{\partial t}+\epsilon \frac{1}{4 \rho} \frac{\partial^{3} V}{\partial x^{2} \partial t}=f(V)-W-\mu V(t-\tau),
$$




$$
\frac{\partial W}{\partial t}=a V-b
$$

The last term of Eq. (20) comparing with Eq. (7) for local variables or with Eq. (14) for global ones was changed after linearizing $g[$ ] in (7) and no threshold $h$. Eq. (21) is taken in simplified form with constant decay. The initial conditions have been given as $V(0)=V(\theta), W(0)=W(\theta)$, where $-\tau \leqslant \theta \leqslant 0$.

It is well known way to go through a stationary state, linearizing approximation and an evaluation of eigenvalues, and to estimate the stability and bifurcation of the task raised. It is remarkable that such way provides necessary and sufficient conditions about behavior of the exact system.

The stationary states of Eqs. (20), (21) are as follows

$$
V_{0}=b / a, \quad W_{0}=\frac{b}{a}\left(-1+d \frac{b}{a}\left(\frac{b}{a}-1\right)\right) .
$$

Let us define a new variables $v=V-V_{0}$ and $w=W-W_{0}$, where $v$ and $w$ measure the perturbations of the equilibrium states $V$ and $W$. Now after introducing new variables and linearizing by Taylor expansion, and transformation of the system Eqs. (20), (21) with new variables to the second order partial differential equation the new equation is as follows

$$
\epsilon \frac{\partial v^{2}}{\partial t^{2}}+\epsilon \frac{1}{4 \rho} \frac{\partial^{4} v}{\partial x^{2} \partial t^{2}}=k_{1} v-a_{1} v+\mu_{1} \frac{v(t-\tau)}{\partial t}
$$

where $k_{1}, a_{1}$, and $\mu_{1}$ have new notations for further convenience. The assumption that the solution of Eqs. (23) is $v=\exp (p(t+x / c))$, where $p$ is an eigenvalue and $c$ is a finite wave propagation speed of an action potential the quasi-polynomial characteristics of eigenvalues of Eq. (24) take place.

The characteristic equation with time-delay is as follow

$$
p^{2}-k p+a-\mu p e^{-p \tau}
$$

where $k=\frac{k_{1}}{\epsilon r}, r=1+\frac{1}{2 \sigma} \frac{x^{2}}{c^{2}}, a=\frac{a_{1}}{\epsilon r}, \mu=\frac{\mu_{1}}{\epsilon r}$ and $k_{1}=-1+d \frac{b}{a}\left(3 \frac{b}{a}-2\right)$.

As the differential Eq. (23) bears delayed variable, the characteristic equation (24) of the stationary solution has an infinite of roots. Thus, the time-delay system (20)-(21) reflects an infinite-dimensional state space. At bifurcation of the stationary state the real parts of roots of the characteristic equation (24) are equal zero and roots are conjugate imaginary, i.e., $p_{ \pm}= \pm i \omega$. Substitution of $p_{ \pm}= \pm i \omega$ into Eq. (24) gives

$$
\omega_{1,2.3 .4}= \pm\left\{-\frac{\left(k^{2}-\mu^{2}-2 a\right)}{2} \pm \frac{1}{2}\left[\left(k^{2}-\mu^{2}-2 a\right)^{2}-4 a^{2}\right]^{1 / 2}\right\}^{1 / 2}
$$

the treatments of which are given in Appendix A.

The critical delay time $\tau_{c}$ is taken (A.4) as follows

$$
\tau \simeq \tau_{c}=\frac{\cos ^{-1}(-k \mu)}{\omega}=\frac{1}{\omega} \arccos (-k / \mu)+2 \pi j,
$$



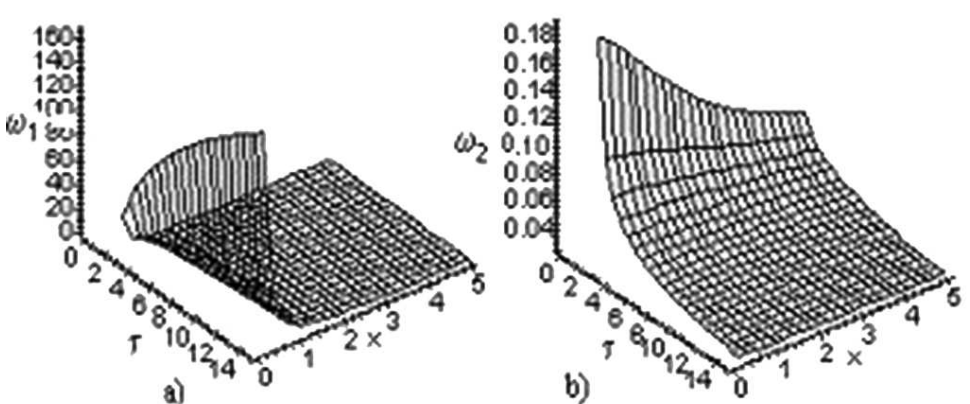

Fig. 8. The frequencies versus delay-time and space coordinate: (a) the frequency $\omega_{1}=\omega_{++}$, (b) the frequency $\omega_{2}=\omega_{+-}$

where $\mu$ is one of four roots according to (A.5). Because of $\omega=2 \pi / T$, between $\omega$ and period $T$ of oscillations occur a such relation

$$
T=\frac{2 \pi}{\omega} .
$$

\subsection{Modeling Results}

To confirm the theoretical backgrounds of an excitable neuronal network dynamics with spatial distribution of the spiking neurons and delay-time in the axon-dendrite channels, an existence of possible bifurcations the modeling of the exposed FitzHugh-Nagumo delaydifferential equations was fulfilled.

The conditions of the bifurcations and stability of the stationary states described by Eqs. (20), (21) were based on some concrete calculations. The changing of the roots of the characteristic equation (24) $\omega_{1}$ and $\omega_{2}$ (see notations in Appendix A) versus parameters $x$ and $\mu$ is shown in Figs. 8(a), 8(b).

The surface in the ranges of parameters (Figs. 8(a), 8(b)) illustrates the region of parametric space, on boundary of which there are periodic solutions of (23) with period given by (27).

Bifurcation curves $\tau_{c, \omega_{1}}$ and $\tau_{c, \omega_{2}}$ for fixed $x, a=0.3, b=0.5, c=0.03, d=1.098$, $j=0$, and $\epsilon=0.001$ are shown in Figs. 9(a), 9(b).

The curves in left-hand side are strictly abrupt at $\mu=3$. It means that an argument of the $\arccos$ becomes more or equal to one or the appropriate angular frequencies $\omega_{1}, \omega_{2}$ become imaginary. The analogous curves $\tau_{c, \omega_{1}}$ for fixed $x=0.1$ and other recent parameters versus cycling number $j$ are shown in Fig. 10(a), as well as for fixed $x=0.1$ and $j=0$ versus parameter $b$, they are shown in Fig. 8b. The conclusion follows that than higher cycling number than the critical values of oscillation behavior more increase.

Thus, when the eigenvalue $p$ is pure imaginary there may be a Hopf bifurcation proportioned to a pair of imaginary conjugate eigenvalues crossing the imaginary axis, dissociating the left- and right hand complex plane. This bifurcation may be either subcritical or supercritical. 

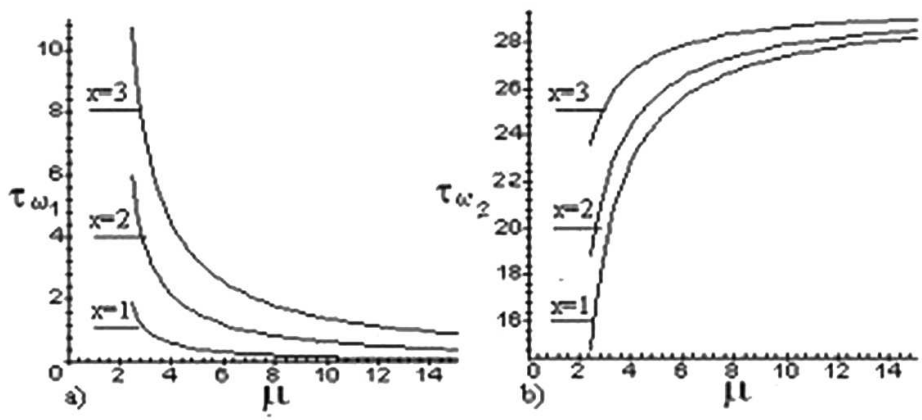

Fig. 9. The critical delay-times versus strength of synapses $\mu$ and space coordinate $x$ : (a) the critical delay-time with $\omega_{1}=\omega_{++}$, (b) the critical delay-time with $\omega_{2}=\omega_{+-}$.
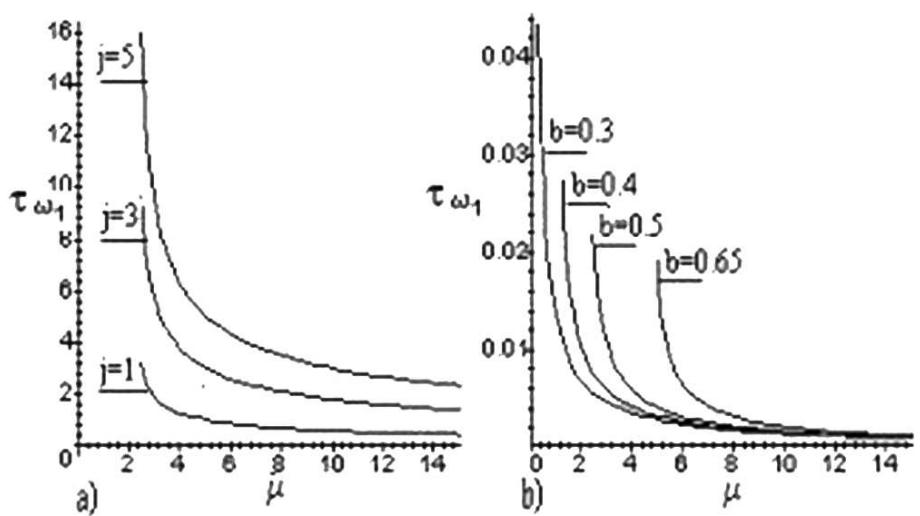

Fig. 10. The critical delay-times versus strength of synapses: (a) at fixed numbers of waves, (b) at fixed decay coefficients of recovery currents.

\section{Conclusions}

In the neural network FitzHugh-Nagumo model, the excited neuronal kernel function was introduced. The introduction of kernel function, which derive to a solution distributed in a space, complicates a search of decision. However, the using of a possibility to present the nonlinear integral differential equations with kernel functions under the Fourier transformation by partial differential equations allowed us to overcome the analytical and numerical modeling difficulties.

The approach of the equivalent partial differential equations was successfully employed to solve the system with the heterogeneous synaptic functions as well as the FitzHugh-Nagumo nonlinear time-delayed differential equations in the case of the Hopf bifurcation and stability of stationary states. In the first time, the necessary and sufficient conditions for the stability of solutions justified by a finding of the critical time-delay have been found depending on the space coordinate. The different numerical modeling experi- 
ments allowed to justify the theoretical foundations in the areas of discovering oscillations, bifurcations, and stability of solutions.

In the future investigations, the local solutions of the neuronal ensembles with noises and time-delays will be studied. We shall include the first and second order cumulants of deviations assuming the Gaussian distribution of the variables and the applications will the subjects of a follow up paper.

\section{Appendix A}

It is necessary to find the roots of the transcendental equation

$$
p^{2}-k p+a-\mu p e^{-p \tau}
$$

in the Hopf bifurcation case when $p=i \omega$.

Substituting $p$ to Eq. (A.1), changing $e^{-i \omega \tau}$ by trigonometrical form, and grouping real parts and imaginary ones one gets

$$
a-\omega^{2}-\mu \omega \sin \omega \tau+i(\mu \omega \cos \omega \tau-k \omega)=0 .
$$

The complex number is equal to zero when the real part and the imaginary one are equal to zero in separately. They are following

$$
\begin{aligned}
& a-\omega^{2}=\mu \omega \sin \omega \tau, \\
& k \omega=\mu \omega \cos \omega \tau
\end{aligned}
$$

after taking quadratics of (A.3) and (A.4) and summarizing all parts of equations the biquadratic equation follows as

$$
\omega^{4}+\left(k^{2}-\mu^{2}-2 a\right) \omega^{2}+a^{2}=0 .
$$

Using standard procedure of solving Eq. (A.5) we get four roots expressed by the form

$$
\omega_{1,2.3 .4}= \pm\left\{-\frac{\left(k^{2}-\mu^{2}-2 a\right)}{2} \pm \frac{1}{2}\left[\left(k^{2}-\mu^{2}-2 a\right)^{2}-4 a^{2}\right]^{1 / 2}\right\}^{1 / 2}
$$

where $\omega_{1}=\omega_{++}, \omega_{2}=\omega_{+-}, \omega_{3}=\omega_{-+}$, and $\omega_{4}=\omega_{--}$.

For finding of a critical delay time $\tau_{c}$, it is used (A.4) and it is got

$$
\tau \simeq \tau_{c}=\frac{\cos ^{-1}(-k \mu)}{\omega}=\frac{1}{\omega} \arccos (-k / \mu)+2 \pi j,
$$

where $\mu$ is one of four roots according to (A.5). Finally, because of $\omega=2 \pi / T$ between $\omega$ and period $T$ of oscillations occur a such relation

$$
T=\frac{2 \pi}{\omega} .
$$




\section{References}

Cowan, J.D. (1961). Spontaneous symmetry breaking in large scale nervous activity. International Journal of Quantum Chemistry, XXII, 1059-1082.

FitzHugh, R. (1961). Impulses and physiological states in theoretical models of nerve membrane. Biophysical Journal, 1, 445 .

Garliauskas, A. (2003). Self-organized topological structures in neural networks for the visual cortex of the brain. Neurocomputing, 51, 13-39.

Garliauskas, A. (1998). Numerical simulation of dynamic synapse-dendrite-soma neuronal processes. Informatica, 9(2), 141-160.

Hale, H., Lamel, S.V. (1993). Introduction of Functional Differential Equations. Springer, New York.

Hasegawa, H. (2003). Augmented moment method for stochastic ensembles with delayed couplings. II. FitzHugh-Nagumo modell. Physical Review E, 68, 041909.

Hasegawa, H. (2004). Dynamical mean field theory of noisy spiking neuron ensembles: application to the Hodgkin-Huxley model. Physical Review E, 68, 041909.

Hodgkin, A.L., Huxley A.F. (1952). A quantitative description of membrane current and its application to conduction and excitation in nerve. Journal of Physiology, 117, 500.

Iooss, G., Joseph, D.D. (1980). Elementary Stability and Bifurcation Theory. Springer, New York.

Hubel, D.H., Wiesel, T.N. (1962). Receptive fields, binocular interaction, and functional architecture in the cat's visual cortex. Journal of Physiology (London), 160, 106-154.

Laing, C.R., Troy, W.C. (2003). PDE methods for nonlocal models. SIAM Journal on Applied Dynamical Systems, 2, 487-516.

Mackey, M.C., Nechaeva, I.G. (1995). Solution moment stability in stochastic delay equations. Physical Review E, 52(4), 3360-3376.

Schindt, P., Crill, W.E. (1977). A persistent negative resistance in cut lumber motoneurons. Brain Research, 120, 173-178.

Szentagothai, J. (1977). The neuron network of the cerebral cortex: a functional interpretation. Proceedings of the Royal Society of London B, 201, 219-248.

Nagumo, J., Arimoto, S., Yoshizawa, S. (1962). An active pulse transmission line simulating nerve axon. Proceedings IRE, 50, 2060-2070.

Tanabe, S., Pakdaman, K. (2001). Dynamics of moments of FitzHugh-Nagumo neuronal models and stochastic bifurcations. Physical Review E, 63, 3191.

Zhang, L., Wu, P.S., Stoner, M.A. (2010). Influence of sodium currents on speeds of traveling wave fronts in synaptically coupled neuronal networks. Physica D, 239, 9-32

A. Garliauskas received his habil. dr. degree of technical sciencies from the Computer Center of the USSR Academy of Sciences, Novosibirsk in 1977. He was a head of the laboratory of Neuroinformatics, Institute of Mathematics and Informatics and a professor of Vilnius University, Vilnius Gedimino Technical University, Mykolas Romeris University. His research interest includes neuroinformaics methodology, modeling neurophysiological processes, chaos, recognition, control and application of neural networks in economy and finances. 
FitzHugh-Hagumo neuroninio tinklo vidutinio lauko su branduolio funkcijomis ir sąveikos laiko delsa modeliavimas

\section{Algis GARLIAUSKAS}

Straipsnyje nagrinėjamas FitzHugh-Nagumo neuroninio tinklo modelis, išplèstas įtraukiant sąveikos branduolio funkciją ir biologiškai natūralią natrio jonų srovès-potencialo charakteristiką. Pasiūlymas transformuoti netiesines integralines diferencialines lygtis pagal Furje ị lygtis su dalinemis išvestinèmis leidžia lengviau įveikti analitinio ir skaitinio modeliavimo trūkumus. Taip pat ištirta FitxHugh-Nagumo netiesinès su laiko delsa diferencialinės lygtys Hoph bifurkacijos ir stacionarių būsenų stabilumo atvejuose. Analitinès studijos paremtos plačiu skaitinio modeliavimo eksperimentu. 